EUROPEAN JOURNAL OF PURE AND APPLIED MATHEMATICS

Vol. 14, No. 4, 2021, 1337-1349

ISSN 1307-5543 - ejpam.com

Published by New York Business Global

\title{
Double integral involving logarithmic and quotient function with powers expressed in terms of the Lerch function
}

\author{
Robert Reynolds ${ }^{1, *}$, Allan Stauffer ${ }^{1}$ \\ 1 Department of Mathematics and Statistics, Faculty of Science, York University, Toronto, \\ Ontario, Canada, M3J1P3
}

\begin{abstract}
In this work the authors use their contour integral method to derive the double integral given by $\int_{0}^{\infty} \int_{0}^{\infty} \frac{x^{m-1} y^{m+\frac{q}{2}-1} \log ^{k}(a x y)}{\left(x^{q}+1\right)^{2}\left(y^{q}+1\right)^{2}} d x d y$ in terms of the Lerch function. This integral formula is then used to derive closed solutions in terms of fundamental constants and special functions. There are some useful results relating double integrals of certain kinds of functions to ordinary integrals for which we know no general reference. Thus a table of integral pairs is given for interested readers. All the results in this work are new.
\end{abstract}

2020 Mathematics Subject Classifications: 30E20, 33-01, 33-03, 33-04, 33-33B, 33E20

Key Words and Phrases: Catalan's constant, Double integral, Apéry's constant, Lerch function, Contour integral

\section{Introduction}

The double integral in terms of the Lerch function derived in this work is used to provide formal derivations and new formulae in the form of a summary table of integrals. The Lerch function being a special function has the fundamental property of analytic continuation, which enables us to widen the range of evaluation for the parameters involved in our definite integral.

The definite integral derived in this manuscript is given by

$$
\int_{0}^{\infty} \int_{0}^{\infty} \frac{x^{m-1} y^{m+\frac{q}{2}-1} \log ^{k}(a x y)}{\left(x^{q}+1\right)^{2}\left(y^{q}+1\right)^{2}} d x d y
$$

where the parameters $k, a$ are general complex numbers and $0<\operatorname{Re}(m)<1$. This work is important because the authors were unable to find similar derivations in current literature.

*Corresponding author.

DOI: https://doi.org/10.29020/nybg.ejpam.v14i4.4085

Email addresses: milver@my.yorku.ca (R. Reynolds), stauffer@yorku.ca (A. Stauffer) 
The derivation of the definite integral follows the method used by us in [3] which involves Cauchy's integral formula. The generalized Cauchy's integral formula is given by

$$
\frac{y^{k}}{\Gamma(k+1)}=\frac{1}{2 \pi i} \int_{C} \frac{e^{w y}}{w^{k+1}} d w .
$$

where $C$ is in general an open contour in the complex plane where the bilinear concomitant has the same value at the end points of the contour. This method involves using a form of equation (2) then multiply both sides by a function, then take a definite integral of both sides. This yields a definite integral in terms of a contour integral. A second contour integral is derived by multiplying equation (2) by a function and performing some substitutions so that the contour integrals are the same.

\section{Definite integral of the contour integral}

We use the method in [3]. The variable of integration in the contour integral is $t=$ $m+w$. The cut and contour are in the first quadrant of the complex $z$-plane. The cut approaches the origin from the interior of the first quadrant and the contour goes round the origin with zero radius and is on opposite sides of the cut. Using equation (2) we replace $y$ by $\log (a x y)$ then multiply by $\frac{x^{m-1} y^{m+\frac{q}{2}-1}}{\left(x^{q}+1\right)^{2}\left(y^{q}+1\right)^{2}}$. Next we take the double infinite integral over $x \in(0, \infty)$ and $y \in(0, \infty)$ to get

$$
\begin{aligned}
& \frac{1}{\Gamma(k+1)} \int_{0}^{\infty} \int_{0}^{\infty} \frac{x^{m-1} y^{m+\frac{q}{2}-1} \log ^{k}(a x y)}{\left(x^{q}+1\right)^{2}\left(y^{q}+1\right)^{2}} d x d y \\
& =\frac{1}{2 \pi i} \int_{0}^{\infty} \int_{0}^{\infty} \int_{C} \frac{a^{w} w^{-k-1} x^{m+w-1} y^{m+\frac{q}{2}+w-1}}{\left(x^{q}+1\right)^{2}\left(y^{q}+1\right)^{2}} d w d x d y \\
& =\frac{1}{2 \pi i} \int_{C} \int_{0}^{\infty} \int_{0}^{\infty} \frac{a^{w} w^{-k-1} x^{m+w-1} y^{m+\frac{q}{2}+w-1}}{\left(x^{q}+1\right)^{2}\left(y^{q}+1\right)^{2}} d x d y d w \\
& =\frac{1}{2 \pi i} \int_{C} \frac{\pi^{2} a^{w} w^{-k-1}(m-q+w)(2 m-q+2 w) \csc \left(\frac{2 \pi(m+w)}{q}\right)}{q^{4}} d w
\end{aligned}
$$

from equation (3.241.5) in [1] where $\operatorname{Re}(w+m)<2 q$. We are able to switch the order of integration over $z, x$ and $y$ using Fubini's theorem since the integrand is of bounded measure over the space $C \times \mathbb{R} \times \mathbb{R}$.

\section{The Lerch function}

We use (9.550) and (9.556) in [1] where $\Phi(z, s, v)$ is the Lerch function which is a generalization of the Hurwitz zeta $\zeta(s, v)$ and Polylogarithm functions $L i_{n}(z)$. The Lerch function has a series representation given by 


$$
\Phi(z, s, v)=\sum_{n=0}^{\infty}(v+n)^{-s} z^{n}
$$

where $|z|<1, v \neq 0,-1, .$. and is continued analytically by its integral representation given by

$$
\Phi(z, s, v)=\frac{1}{\Gamma(s)} \int_{0}^{\infty} \frac{t^{s-1} e^{-v t}}{1-z e^{-t}} d t=\frac{1}{\Gamma(s)} \int_{0}^{\infty} \frac{t^{s-1} e^{-(v-1) t}}{e^{t}-z} d t
$$

where $\operatorname{Re}(v)>0$, and either $|z| \leq 1, z \neq 1, \operatorname{Re}(s)>0$, or $z=1, \operatorname{Re}(s)>1$.

\section{Infinite sum of the contour integral}

\subsection{Derivation of the first contour integral}

In this section we will again use Cauchy's integral formula (2) and taking the infinite sum to derive equivalent sum representations for the contour integrals. We proceed using equation (2) and replace $y$ by $\log (a)+\frac{2 i \pi(2 y+1)}{q}$ and multiply both sides by $-\frac{4 i \pi^{2} m^{2} e^{\frac{2 i \pi m(2 y+1)}{q}}}{q^{4}}$ and take the infinite sum over $y \in[0, \infty)$ simplifying in terms of the Lerch function to get

$$
\begin{aligned}
& \frac{4^{k+1} \pi^{k+2} m^{2}\left(\frac{i}{q}\right)^{k-1} e^{\frac{2 i \pi m}{q}} \Phi\left(e^{\frac{4 i m \pi}{q}},-k, \frac{1}{2}-\frac{i q \log (a)}{4 \pi}\right)}{q^{5} \Gamma(k+1)} \\
& =-\frac{1}{2 \pi i} \sum_{y=0}^{\infty} \int_{C} \frac{4 i \pi^{2} m^{2} a^{w} w^{-k-1} e^{\frac{2 i \pi(2 y+1)(m+w)}{q}}}{q^{4}} d w \\
& =-\frac{1}{2 \pi i} \int_{C} \sum_{y=0}^{\infty} \frac{4 i \pi^{2} m^{2} a^{w} w^{-k-1} e^{\frac{2 i \pi(2 y+1)(m+w)}{q}}}{q^{4}} d w \\
& =\frac{1}{2 \pi i} \int_{C} \frac{2 \pi^{2} m^{2} a^{w} w^{-k-1} \csc \left(\frac{2 \pi(m+w)}{q}\right)}{q^{4}} d w
\end{aligned}
$$

from equation (1.232.3) in [1] where $\operatorname{Im}(m+w)>0$ for convergence of the sum.

\subsection{Derivation of the second contour integral}

In this section we will again use Cauchy's integral formula (2) and taking the infinite sum to derive equivalent sum representations for the contour integrals. We proceed using equation (2) and replace $y$ by $\log (a)+\frac{2 i \pi(2 y+1)}{q}$ and multiply both sides by $\frac{6 i \pi^{2} m e^{\frac{2 i \pi m(2 y+1)}{q}}}{q^{3}}$ and take the infinite sum over $y \in[0, \infty)$ simplifying in terms of the Lerch function to get 


$$
\begin{aligned}
& \frac{3 i 2^{2 k+1} \pi^{k+2} m\left(\frac{i}{q}\right)^{k} e^{\frac{2 i \pi m}{q}} \Phi\left(e^{\frac{4 i m \pi}{q}},-k, \frac{1}{2}-\frac{i q \log (a)}{4 \pi}\right)}{q^{3} \Gamma(k+1)} \\
& =\frac{1}{2 \pi i} \sum_{y=0}^{\infty} \int_{C} \frac{6 i \pi^{2} m a^{w} w^{-k-1} e^{\frac{2 i \pi(2 y+1)(m+w)}{q}}}{q^{3}} d w \\
& =\frac{1}{2 \pi i} \int_{C} \sum_{y=0}^{\infty} \frac{6 i \pi^{2} m a^{w} w^{-k-1} e^{\frac{2 i \pi(2 y+1)(m+w)}{q}}}{q^{3}} d w \\
& =-\frac{1}{2 \pi i} \int_{C} \frac{3 \pi^{2} m a^{w} w^{-k-1} \csc \left(\frac{2 \pi(m+w)}{q}\right)}{q^{3}} d w
\end{aligned}
$$

from equation (1.232.3) in [1] where $\operatorname{Im}(m+w)>0$ for convergence of the sum.

\subsection{Derivation of the third contour integral}

In this section we will again use Cauchy's integral formula (2) and taking the infinite sum to derive equivalent sum representations for the contour integrals. We proceed using equation (2) and replace $y$ by $\log (a)+\frac{2 i \pi(2 y+1)}{q}$ and multiply both sides by $-\frac{2 i \pi^{2} e^{\frac{2 i \pi m(2 y+1)}{q}}}{q^{2}}$ and take the infinite sum over $y \in[0, \infty)$ simplifying in terms of the Lerch function to get

$$
\begin{aligned}
& \frac{2^{2 k+1} \pi^{k+2}\left(\frac{i}{q}\right)^{k-1} e^{\frac{2 i \pi m}{q}} \Phi\left(e^{\frac{4 i m \pi}{q}},-k, \frac{1}{2}-\frac{i q \log (a)}{4 \pi}\right)}{q^{3} \Gamma(k+1)} \\
& =-\frac{1}{2 \pi i} \sum_{y=0}^{\infty} \int_{C} \frac{2 i \pi^{2} a^{w} w^{-k-1} e^{\frac{2 i \pi(2 y+1)(m+w)}{q}}}{q^{2}} d w \\
& =-\frac{1}{2 \pi i} \int_{C} \sum_{y=0}^{\infty} \frac{2 i \pi^{2} a^{w} w^{-k-1} e^{\frac{2 i \pi(2 y+1)(m+w)}{q}}}{q^{2}} d w \\
& =\frac{1}{2 \pi i} \int_{C} \frac{\pi^{2} a^{w} w^{-k-1} \csc \left(\frac{2 \pi(m+w)}{q}\right)}{q^{2}} d w
\end{aligned}
$$

from equation (1.232.3) in [1] where $\operatorname{Im}(m+w)>0$ for convergence of the sum.

\subsection{Derivation of the fourth contour integral}

In this section we will again use Cauchy's integral formula (2) and taking the infinite sum to derive equivalent sum representations for the contour integrals. We proceed using equation (2) and replace $y$ by $\log (a)+\frac{2 i \pi(2 y+1)}{q}$ and multiply both sides by $-\frac{4 i \pi^{2} e^{\frac{2 i \pi m(2 y+1)}{q}}}{q^{4}}$ and replace $k \rightarrow k-2$ and take the infinite sum over $y \in[0, \infty)$ simplifying in terms of the Lerch function to get 


$$
\begin{aligned}
& \frac{4^{k-1} \pi^{k}\left(\frac{i}{q}\right)^{k-3} e^{\frac{2 i \pi m}{q}} \Phi\left(e^{\frac{4 i m \pi}{q}}, 2-k, \frac{1}{2}-\frac{i q \log (a)}{4 \pi}\right)}{q^{5} \Gamma(k-1)} \\
& =-\frac{1}{2 \pi i} \sum_{y=0}^{\infty} \int_{C} \frac{4 i \pi^{2} a^{w} w^{1-k} e^{\frac{2 i \pi(2 y+1)(m+w)}{q}}}{q^{4}} d w \\
& =-\frac{1}{2 \pi i} \int_{C} \sum_{y=0}^{\infty} \frac{4 i \pi^{2} a^{w} w^{1-k} e^{\frac{2 i \pi(2 y+1)(m+w)}{q}}}{q^{4}} d w \\
& =\frac{1}{2 \pi i} \int_{C} \frac{2 \pi^{2} a^{w} w^{1-k} \csc \left(\frac{2 \pi(m+w)}{q}\right)}{q^{4}} d w
\end{aligned}
$$

from equation (1.232.3) in [1] where $\operatorname{Im}(m+w)>0$ for convergence of the sum.

\subsection{Derivation of the fifth contour integral}

In this section we will again use Cauchy's integral formula (2) and taking the infinite sum to derive equivalent sum representations for the contour integrals. We proceed using equation (2) and replace $y$ by $\log (a)+\frac{2 i \pi(2 y+1)}{q}$ and multiply both sides by $-\frac{4 i \pi^{2} e^{\frac{2 i \pi m(2 y+1)}{q}}}{q^{4}}$ and replace $k \rightarrow k-1$ and take the infinite sum over $y \in[0, \infty)$ simplifying in terms of the Lerch function to get

$$
\begin{aligned}
& \frac{2^{2(k-1)+3} \pi^{k+1} m\left(\frac{i}{q}\right)^{k-2} e^{\frac{2 i \pi m}{q}} \Phi\left(e^{\frac{4 i m \pi}{q}}, 1-k, \frac{1}{2}-\frac{i q \log (a)}{4 \pi}\right)}{q^{5} \Gamma(k)} \\
& =-\frac{1}{2 \pi i} \sum_{y=0}^{\infty} \int_{C} \frac{8 i \pi^{2} m a^{w} w^{-k} e^{\frac{2 i \pi(2 y+1)(m+w)}{q}}}{q^{4}} d w \\
& =-\frac{1}{2 \pi i} \int_{C} \sum_{y=0}^{\infty} \frac{8 i \pi^{2} m a^{w} w^{-k} e^{\frac{2 i \pi(2 y+1)(m+w)}{q}}}{q^{4}} d w \\
& =\frac{1}{2 \pi i} \int_{C} \frac{4 \pi^{2} m a^{w} w^{-k} \csc \left(\frac{2 \pi(m+w)}{q}\right)}{q^{4}} d w
\end{aligned}
$$

from equation (1.232.3) in [1] where $\operatorname{Im}(m+w)>0$ for convergence of the sum.

\subsection{Derivation of the sixth contour integral}

In this section we will again use Cauchy's integral formula (2) and taking the infinite sum to derive equivalent sum representations for the contour integrals. We proceed using equation $(2)$ and replace $y$ by $\log (a)+\frac{2 i \pi(2 y+1)}{q}$ and multiply both sides by $\frac{6 i \pi^{2} e^{\frac{2 i \pi m(2 y+1)}{q}}}{q^{3}}$ 
and replace $k \rightarrow k-1$ and take the infinite sum over $y \in[0, \infty)$ simplifying in terms of the Lerch function to get

$$
\begin{aligned}
& \frac{3 i 2^{2(k-1)+1} \pi^{k+1}\left(\frac{i}{q}\right)^{k-1} e^{\frac{2 i \pi m}{q}} \Phi\left(e^{\frac{4 i m \pi}{q}}, 1-k, \frac{1}{2}-\frac{i q \log (a)}{4 \pi}\right)}{q^{3} \Gamma(k)} \\
& =\frac{1}{2 \pi i} \sum_{y=0}^{\infty} \int_{C} \frac{6 i \pi^{2} a^{w} w^{-k} e^{\frac{2 i \pi(2 y+1)(m+w)}{q}}}{q^{3}} d w \\
& =\frac{1}{2 \pi i} \int_{C} \sum_{y=0}^{\infty} \frac{6 i \pi^{2} a^{w} w^{-k} e^{\frac{2 i \pi(2 y+1)(m+w)}{q}}}{q^{3}} d w \\
& =-\frac{1}{2 \pi i} \int_{C} \frac{3 \pi^{2} a^{w} w^{-k} \csc \left(\frac{2 \pi(m+w)}{q}\right)}{q^{3}} d w
\end{aligned}
$$

from equation (1.232.3) in [1] where $\operatorname{Im}(m+w)>0$ for convergence of the sum.

\section{Main results}

\section{Definite integral in terms of the Lerch function}

Theorem 1. For $a, k \in \mathbb{C}, 0<\operatorname{Re}(m)<1, \operatorname{Re}(q)>0$,

$$
\begin{aligned}
\int_{0}^{\infty} \int_{0}^{\infty} \frac{x^{m-1} y^{m+\frac{q}{2}-1} \log ^{k}(a x y)}{\left(x^{q}+1\right)^{2}\left(y^{q}+1\right)^{2}} d x d y & \\
& =\frac{4^{k-1} \pi^{k}}{q^{4}}\left(\frac{i}{q}\right)^{k} \\
& e^{\frac{2 i \pi m}{q}}\left(i \left((k-1) k q^{2} \Phi\left(e^{\frac{4 i m \pi}{q}}, 2-k, \frac{1}{2}-\frac{i q \log (a)}{4 \pi}\right)\right.\right. \\
& \left.-8 \pi^{2}(q-2 m)(q-m) \Phi\left(e^{\frac{4 i m \pi}{q}},-k, \frac{1}{2}-\frac{i q \log (a)}{4 \pi}\right)\right) \\
& \left.+2 \pi k q(3 q-4 m) \Phi\left(e^{\frac{4 i m \pi}{q}}, 1-k, \frac{1}{2}-\frac{i q \log (a)}{4 \pi}\right)\right)
\end{aligned}
$$

Proof. Since the addition of the right-hand sides of equations (6), (7), (8), (9), (10) and (11) is equal to the right-hand side of equation (3) we can equate the left-hand sides and reduce the factorial to get the stated result. 
R. Reynolds, A. Stauffer / Eur. J. Pure Appl. Math, 14 (4) (2021), 1337-1349

6. Integral representation for Catalan's constant $G$, Apéry's constant $\zeta(3)$ and $\pi$

\section{Proposition 1.}

$$
\int_{0}^{\infty} \int_{0}^{\infty} \frac{\sqrt[4]{x}(\sqrt{x} \sqrt{y}-1)}{(x+1)^{2} \sqrt[4]{y}(y+1)^{2} \log (x y)} d x d y=G
$$

Proof. Use (12) and form a second equation by replacing $m \rightarrow p$ and take their difference. Then set $k=-1, a=1, q=-1, m=-3 / 4, p=-1 / 4$ and simplify using entries (1), (2), (4) in Table below (64:12:7) in [2].

\section{Proposition 2.}

$$
\int_{0}^{\infty} \int_{0}^{\infty} \frac{\sqrt[4]{x} \sqrt[4]{y}-1}{x^{3 / 4}(x+1)^{2} \sqrt[4]{y}(y+1)^{2} \log (x y)} d x d y=G-\frac{7 \zeta(3)}{8 \pi}
$$

Proof. Use (12) and form a second equation by replacing $m \rightarrow p$ and take their difference. Then set $k=-1, a=1, m=1 / 4, p=1 / 2$. Then apply L'Hopital's rule to the right-hand side as $q \rightarrow 1$ and simplify using entries (1), (2), (4) in Table below (64:12:7), equation (64:12:1) and entry (2) in Table below (64:7) in [2].

\section{Proposition 3.}

$$
\int_{0}^{\infty} \int_{0}^{\infty} \frac{y^{2}\left(1-x^{2} y^{2}\right) \log (x y)}{\left(x^{4}+1\right)^{2}\left(y^{4}+1\right)^{2}\left(\log ^{2}(x y)+\pi^{2}\right)} d x d y=\frac{1}{4}(G-1)
$$

and

$$
\int_{0}^{\infty} \int_{0}^{\infty} \frac{y^{2}\left(x^{2} y^{2}-1\right)}{\left(x^{4}+1\right)^{2}\left(y^{4}+1\right)^{2}\left(\log ^{2}(x y)+\pi^{2}\right)} d x d y=\frac{1}{2 \pi^{2}}-\frac{1}{16}
$$

Proof. Use (12) and form a second equation by replacing $m \rightarrow p$ and take their difference. Then set $k=-1, a=-1, q=4, m=3, p=1$ rationalize the denominator and equate real and imaginary parts and simplify using entries (1), (2), (4) in Table below $(64: 12: 7)$ in [2]. 


\section{Definite integral in terms of the polylogarithm function}

Theorem 2. For $k \in \mathbb{C}, 0<\operatorname{Re}(m)<1, \operatorname{Re}(q)>0$,

$$
\begin{aligned}
& \int_{0}^{\infty} \int_{0}^{\infty} \frac{x^{m-1} y^{m+\frac{q}{2}-1} \log ^{k}\left(e^{\frac{2 i \pi}{q}} x y\right)}{\left(x^{q}+1\right)^{2}\left(y^{q}+1\right)^{2}} d x d y \\
& =\frac{4^{k-1} \pi^{k}}{q^{4}}\left(\frac{i}{q}\right)^{k} \\
& \quad e^{-\frac{2 i \pi m}{q}}\left(i \left((k-1) k q^{2} L i_{2-k}\left(e^{\frac{4 i m \pi}{q}}\right)\right.\right. \\
& \left.\quad-8 \pi^{2}(q-2 m)(q-m) L i_{-k}\left(e^{\frac{4 i m \pi}{q}}\right)\right) \\
& \left.+2 \pi k q(3 q-4 m) L i_{1-k}\left(e^{\frac{4 i m \pi}{q}}\right)\right)
\end{aligned}
$$

Proof. Use (12) and replace $a \rightarrow e^{\frac{2 \pi i}{q}}$ and simplify using equation (64:12:2) in [2].

\section{Definite integral in terms of the Hurwitz zeta function}

Theorem 3. For $k \in \mathbb{C}, \operatorname{Re}(q)>0$,

$$
\begin{aligned}
& \int_{0}^{\infty} \int_{0}^{\infty} \frac{x^{\frac{q}{4}-1} y^{\frac{3 q}{4}-1} \log ^{k}(x y)}{\left(x^{q}+1\right)^{2}\left(y^{q}+1\right)^{2}} d x d y \\
& =\frac{2^{3 k-4} \pi^{k}}{q^{2}}\left(8 i \pi k \zeta\left(1-k, \frac{1}{4}\right)\right. \\
& \quad-8 i \pi k \zeta\left(1-k, \frac{3}{4}\right) \\
& \quad-(k-1) k\left(\zeta\left(2-k, \frac{1}{4}\right)\right. \\
& \left.\quad-\zeta\left(2-k, \frac{3}{4}\right)\right) \\
& +12 \pi^{2}\left(\zeta\left(-k, \frac{1}{4}\right)\right. \\
& \left.\left.\quad-\zeta\left(-k, \frac{3}{4}\right)\right)\right) \\
& \left(\frac{i}{q}\right)^{k}
\end{aligned}
$$

Proof. Use (12) set $a=1$ and replace $m \rightarrow q / 4$ and simplify using equation (64:12:1) and entry (4) in Table below (64:12:7) in [2]. 


\section{Derivation of entry 3.241 .5 in [1]}

Proposition 4. For $0<\operatorname{Re}(m)<1,0<\operatorname{Re}(p)<1, \operatorname{Re}(p)<2 q$,

$$
\begin{aligned}
\int_{0}^{\infty} \int_{0}^{\infty} \frac{y^{\frac{q}{2}-1}\left(x^{p} y^{p}-x^{m} y^{m}\right)}{x\left(x^{q}+1\right)^{2}\left(y^{q}+1\right)^{2}} d x d y \\
=\frac{\pi^{2}\left((2 m-q)(q-m) \csc \left(\frac{2 \pi m}{q}\right)+(q-2 p)(q-p) \csc \left(\frac{2 \pi p}{q}\right)\right)}{q^{4}}
\end{aligned}
$$

Proof. Use equation (12) and set $k=0$ and simplify using entry (2) in Table below $(64: 12: 7)$ in $[2]$.

Proposition 5.

$$
\begin{aligned}
& \int_{0}^{\infty} \int_{0}^{\infty} \frac{\sqrt{y} \log (\log (x y))}{\sqrt{x}\left(x^{2}+1\right)^{2}\left(y^{2}+1\right)^{2}} d x d y \\
& =\frac{1}{64}\left(16 G+\pi^{2}\left(8 i+3 i \pi+2 \log \left(\frac{64 \pi^{3} \Gamma\left(-\frac{1}{4}\right)^{6}}{729 \Gamma\left(-\frac{3}{4}\right)^{6}}\right)\right)\right)
\end{aligned}
$$

Proof. Use equation (18) and take the first partial derivative with respect to $k$ and set $k=0, q=2$ and simplify using equation $(64: 10: 2)$ in [2].

\section{Proposition 6.}

$$
\begin{aligned}
& \int_{0}^{\infty} \int_{0}^{\infty} \frac{\sqrt{y} \log (x y) \log (\log (x y))}{\sqrt{x}\left(x^{2}+1\right)^{2}\left(y^{2}+1\right)^{2}} d x d y \\
& \quad=\frac{1}{16} \pi^{2}\left(-6 i G+(-4-i)-2 i \pi+\log \left(\frac{6561 \Gamma\left(-\frac{3}{4}\right)^{8}}{256 \pi^{4} \Gamma\left(-\frac{1}{4}\right)^{8}}\right)\right)
\end{aligned}
$$

Proof. Use equation (18) and apply L'Hopital's rule as $k \rightarrow 1$ and set $q=2$ and simplify using equation $(64: 10: 2)$ in [2].

\section{Definite integral in terms of the Zeta function of Riemann}

\section{Lemma 1.}

$$
\begin{aligned}
& \int_{0}^{\infty} \int_{0}^{\infty} \frac{\sqrt{y}}{\sqrt{x}\left(x^{2}+1\right)^{2}\left(y^{2}+1\right)^{2}\left(\log ^{2}(x y)+\pi^{2}\right)} d x d y \\
& =\frac{3}{32}\left(2 \zeta^{\prime}(-2)+\log (2)\right)
\end{aligned}
$$


Proof. Use equation (12) and set $m=1 / 2, a=-1, q=2$ and simplify in terms of the Riemann zeta function using entry (2) in Tbale below (64:7) and entry (4) in table below $(64: 12: 7)$ in $[2]$ to get

$$
\begin{aligned}
\int_{0}^{\infty} & \int_{0}^{\infty} \frac{\sqrt{y} \log ^{k}(-x y)}{\sqrt{x}\left(x^{2}+1\right)^{2}\left(y^{2}+1\right)^{2}} d x d y \\
& =2^{k-5}(i \pi)^{k}\left(-8 i \pi\left(2^{k}-1\right)\right. \\
& k \zeta(1-k)+\left(2^{k}-2\right) \\
& \left.(k-1) k \zeta(2-k)-6 \pi^{2}\left(2^{k+1}-1\right) \zeta(-k)\right)
\end{aligned}
$$

Next apply L'Hopital's rule as $k \rightarrow-1$ and simplify.

\section{Proposition 7.}

$$
\begin{aligned}
& \int_{0}^{\infty} \int_{0}^{\infty} \frac{\sqrt{y}}{\sqrt{x}\left(x^{2}+1\right)^{2}\left(y^{2}+1\right)^{2}\left(\log ^{2}(x y)+\pi^{2}\right)} d x d y \\
& =\frac{3}{32}\left(2 \zeta^{\prime}(-2)+\log (2)\right)
\end{aligned}
$$

and

$$
\int_{0}^{\infty} \int_{0}^{\infty} \frac{\sqrt{y} \log (x y)}{\sqrt{x}\left(x^{2}+1\right)^{2}\left(y^{2}+1\right)^{2}\left(\log ^{2}(x y)+\pi^{2}\right)} d x d y=-\frac{\pi^{2}}{96}
$$

Proof. Use equation (23) and apply L'Hopitals' rule as $k \rightarrow-1$ rationalize the denominator and equate real and imaginary parts to get stated result.

Theorem 4. For $k \in \mathbb{C}$,

$$
\begin{aligned}
\int_{0}^{\infty} & \int_{0}^{\infty} \frac{y^{2} \log ^{k}(i x y)}{\left(x^{4}+1\right)^{2}\left(y^{4}+1\right)^{2}} d x d y \\
= & \frac{1}{128}(i \pi)^{k}\left(-8 i \pi\left(2^{k}-1\right)\right. \\
& k \zeta(1-k)+\left(2^{k}-2\right) \\
& \left.(k-1) k \zeta(2-k)-6 \pi^{2}\left(2^{k+1}-1\right) \zeta(-k)\right)
\end{aligned}
$$

Proof. Use (12) and set $m=1, q=4, a=i$ and simplify using entry (4) in Table below $(64: 12: 7)$ in $[2]$. 
Proposition 8.

$$
\begin{aligned}
& \int_{0}^{\infty} \int_{0}^{\infty} \frac{y^{2}}{\left(x^{4}+1\right)^{2}\left(y^{4}+1\right)^{2}\left(4 \log ^{2}(x y)+\pi^{2}\right)} d x d y \\
& =\frac{3}{128}\left(2 \zeta^{\prime}(-2)+\log (2)\right)
\end{aligned}
$$

and

$$
\int_{0}^{\infty} \int_{0}^{\infty} \frac{y^{2} \log (x y)}{\left(x^{4}+1\right)^{2}\left(y^{4}+1\right)^{2}\left(4 \log ^{2}(x y)+\pi^{2}\right)} d x d y=-\frac{\pi^{2}}{768}
$$

Proof. Use equation (26) and apply L'Hopitals' rule as $k \rightarrow-1$ rationalize the denominator and equate real and imaginary parts to get stated result. 


\section{Summary table of results}

$$
\begin{aligned}
& f(x, y) \quad \int_{0}^{\infty} \int_{0}^{\infty} f(x, y) d x d y \\
& \frac{\sqrt[4]{x}(\sqrt{x} \sqrt{y}-1)}{(x+1)^{2} \sqrt[4]{y}(y+1)^{2} \log (x y)} \\
& G \\
& \frac{\sqrt[4]{x} \sqrt[4]{y}-1}{x^{3 / 4}(x+1)^{2} \sqrt[4]{y}(y+1)^{2} \log (x y)} \\
& G-\frac{7 \zeta(3)}{8 \pi} \\
& \frac{y^{2}\left(x^{2} y^{2}-1\right)}{\left(x^{4}+1\right)^{2}\left(y^{4}+1\right)^{2}\left(\log ^{2}(x y)+\pi^{2}\right)} \\
& \frac{1}{2 \pi^{2}}-\frac{1}{16} \\
& \frac{y^{2}\left(1-x^{2} y^{2}\right) \log (x y)}{\left(x^{4}+1\right)^{2}\left(y^{4}+1\right)^{2}\left(\log ^{2}(x y)+\pi^{2}\right)} \\
& \frac{1}{4}(G-1) \\
& \frac{y^{\frac{q}{2}-1}\left(x^{p} y^{p}-x^{m} y^{m}\right)}{x\left(x^{q}+1\right)^{2}\left(y^{q}+1\right)^{2}} \\
& \frac{\pi^{2}\left((2 m-q)(q-m) \csc \left(\frac{2 \pi m}{q}\right)+(q-2 p)(q-p) \csc \left(\frac{2 \pi p}{q}\right)\right)}{q^{4}} \\
& \frac{\sqrt{y} \log (\log (x y))}{\sqrt{x}\left(x^{2}+1\right)^{2}\left(y^{2}+1\right)^{2}} \\
& \frac{1}{64}\left(16 G+\pi^{2}\left(8 i+3 i \pi+2 \log \left(\frac{64 \pi^{3} \Gamma\left(-\frac{1}{4}\right)^{6}}{729 \Gamma\left(-\frac{3}{4}\right)^{6}}\right)\right)\right) \\
& \frac{\sqrt{y} \log (x y) \log (\log (x y))}{\sqrt{x}\left(x^{2}+1\right)^{2}\left(y^{2}+1\right)^{2}} \\
& \frac{1}{16} \pi^{2}\left(-6 i G+(-4-i)-2 i \pi+\log \left(\frac{6561 \Gamma\left(-\frac{3}{4}\right)^{8}}{256 \pi^{4} \Gamma\left(-\frac{1}{4}\right)^{8}}\right)\right) \\
& \frac{\sqrt{y}}{\sqrt{x}\left(x^{2}+1\right)^{2}\left(y^{2}+1\right)^{2}\left(\log ^{2}(x y)+\pi^{2}\right)} \\
& \frac{3}{32}\left(2 \zeta^{\prime}(-2)+\log (2)\right) \\
& \frac{\sqrt{y} \log (x y)}{\sqrt{x}\left(x^{2}+1\right)^{2}\left(y^{2}+1\right)^{2}\left(\log ^{2}(x y)+\pi^{2}\right)} \\
& -\frac{\pi^{2}}{96} \\
& \frac{y^{2}}{\left(x^{4}+1\right)^{2}\left(y^{4}+1\right)^{2}\left(4 \log ^{2}(x y)+\pi^{2}\right)} \\
& \frac{3}{128}\left(2 \zeta^{\prime}(-2)+\log (2)\right) \\
& \frac{y^{2} \log (x y)}{\left(x^{4}+1\right)^{2}\left(y^{4}+1\right)^{2}\left(4 \log ^{2}(x y)+\pi^{2}\right)} \\
& -\frac{\pi^{2}}{768}
\end{aligned}
$$

\section{Discussion}

In this work the authors derived a double integral formula in terms of the Lerch function. This integral formula was then used to derive special cases in terms of fundamental constants and special functions. A table of integrals featuring some of the integral results was presented for the benefit of interested readers. We used Wolfram Mathematica to 
numerically verify the formulas for various ranges of the parameters for real and imaginary values. We will use our contour integral method to derive other double integrals and produce more tables of integrals in our future work.

\section{References}

[1] I. S. Gradshteyn and I. M. Ryzhik. Table of Integrals, Series, and Products. Academic Press, 052014.

[2] Keith B. Oldham, Jan Myland, and Jerome Spanier. An Atlas of Functions: with Equator, the Atlas Function Calculator. Springer Science \& Business Media, 072010.

[3] Robert Reynolds and Allan Stauffer. A method for evaluating definite integrals in terms of special functions with examples. International Mathematical Forum, 15:235$244,2020$. 